PROCEEDINGS OF THE

AMERICAN MATHEMATICAL SOCIETY

Volume 125, Number 2, February 1997, Pages 617-623

S 0002-9939(97)03760-X

\title{
NORMAL EULER CLASSES OF KNOTTED SURFACES AND TRIPLE POINTS ON PROJECTIONS
}

\author{
J. SCOTT CARTER AND MASAHICO SAITO
}

(Communicated by Ronald Stern)

\begin{abstract}
We present a new formula relating the normal Euler numbers of embedded surfaces in 4-space and the number of triple points on their projections into 3-space. This formula generalizes Banchoff's formula between normal Euler numbers and branch points on the projections.
\end{abstract}

\section{INTRODUCTION}

A formula between normal Euler numbers of embedded surfaces in 4-space and the number of branch points on their projections into 3-space was discovered by Banchoff [1] (see also [2]). In this paper we generalize his formula including the number of triple points on projections. For the rest of the paper the term knotted surfaces refers to smoothly embedded closed surfaces in four-dimensional Euclidean space. We use the term knotted surfaces since the phenomena of knottings occur for codimension 2 embeddings, and the results of the present paper arose from our studies in knot theory in 4-space [2,3].

The normal Euler number is calculated as follows [2]. Let $F$ be a knotted surface in 4-space. Let $F_{1}$ be a transverse push-off of $F$. Thus $F \cap F_{1}$ consists of isolated transverse double intersection points. At each point of $F \cap F_{1}$, choose a local orientation of $F$ and an induced orientation of $F_{1}$. The local intersection number between these surfaces is computed by comparing the orientation of the two intersecting surfaces with a fixed orientation of $\mathbf{R}^{4}$. If the orientations agree (resp. do not agree), then the point contributes +1 (resp. -1) to the normal Euler number. Thus the normal Euler number $e(F)$ is the sum of these intersection numbers taken over all the points of $F \cap F_{1}$. The normal Euler number is, in fact, a characteristic class of the normal bundle of the embedding of the surface in 4-space.

Banchoff's formula relates the normal Euler numbers to branch points on the projections. A generic projection of a knotted surface in 4-space into 3-space consists of neighborhoods of (1) embedded points, (2) double point arcs, (3) isolated triple points, and (4) branch points $[5,2,3]$. The double point curve (resp. a triple point, a branch point) are depicted in the left of Figure 1 (A) (resp. (B), (C)). If $F$ is a knotted surface, then it can be assumed without loss of generality that

Received by the editors July 27, 1995.

1991 Mathematics Subject Classification. Primary 57Q45.

Key words and phrases. Knotted surfaces, normal Euler numbers, checker-board coloring, triple points, branch points.

Presented at the 872nd meeting of the AMS, Tuscaloosa.

(C)1997 American Mathematical Society 


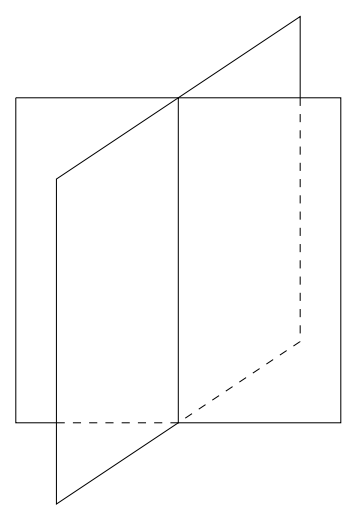

( A )
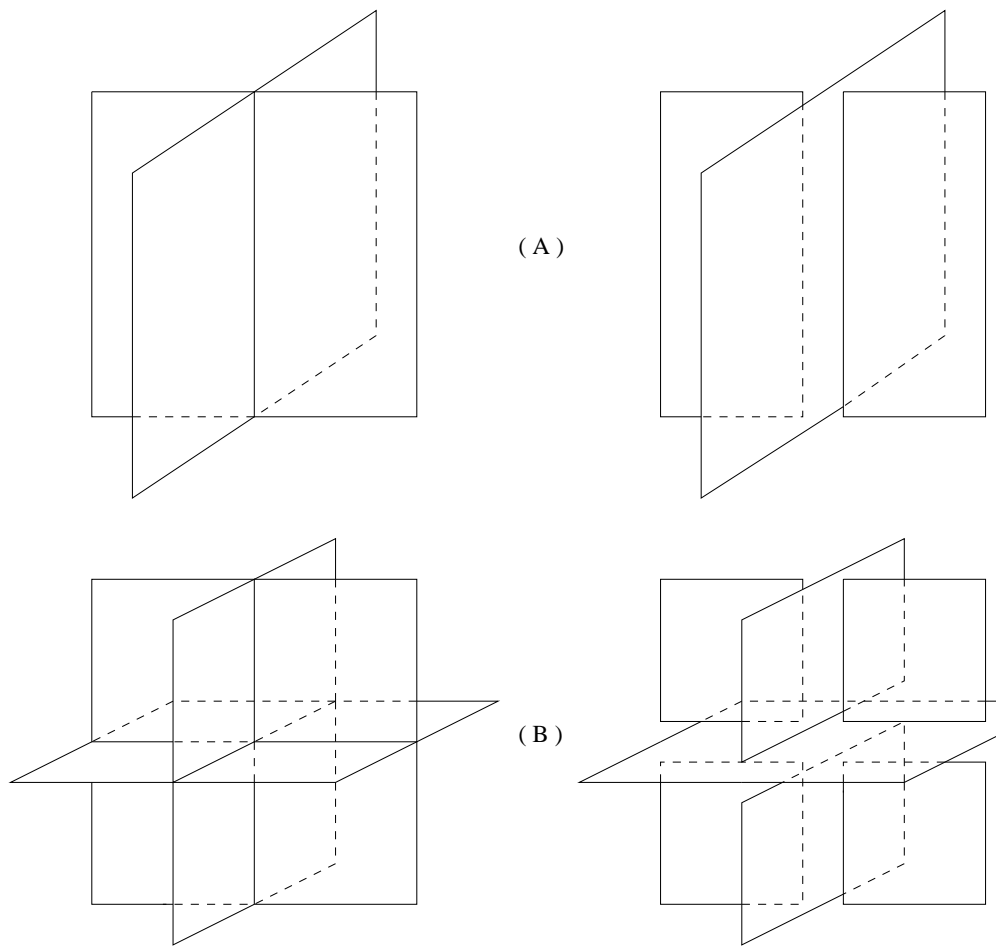

( B )
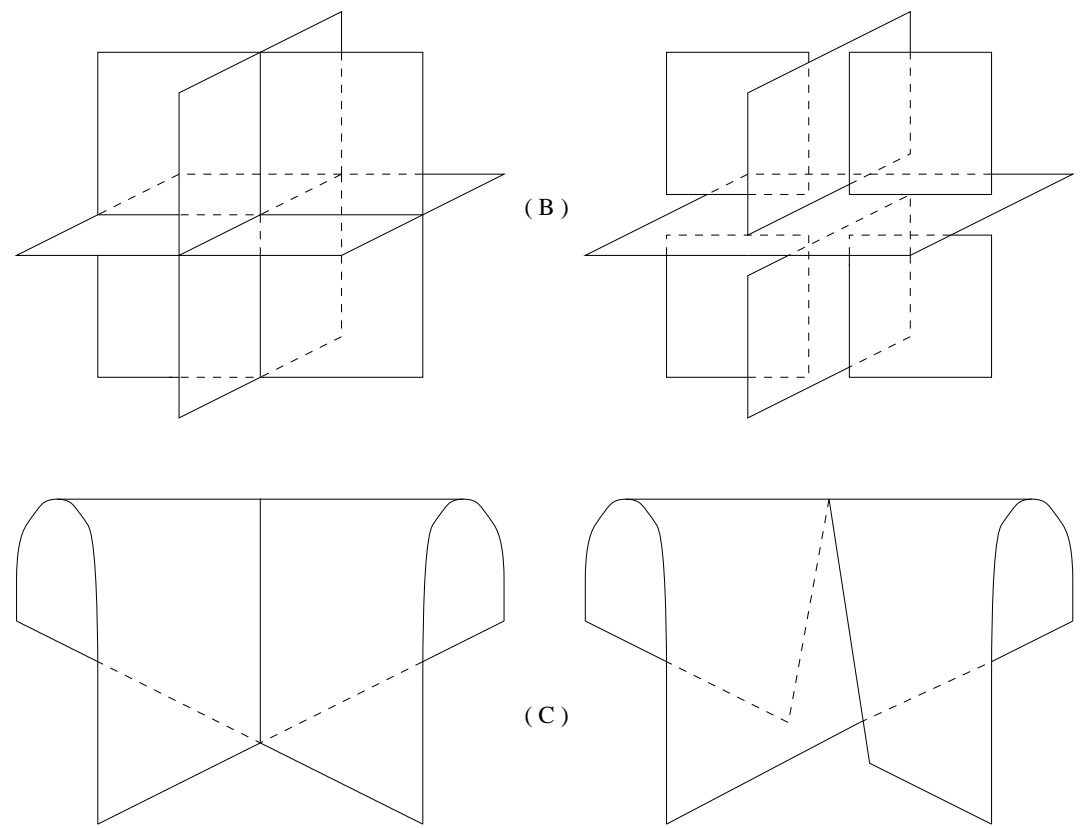

FiguRE 1. Projections and broken diagrams of knotted surfaces

the image $p(F)$ under the projection $p: \mathbf{R}^{4} \rightarrow \mathbf{R}^{3}$ into a 3-dimensional subspace is such a generic surface. Furthermore, along double point curves along which two sheets intersect, there is crossing information; namely which of the two sheets along a double curve is closer to the hyperplane of projection than the other. We use broken surface diagrams to indicate the crossing information [3]. Specifically, we break the neighborhood of the double point set of the sheet which lies further away from the projectional hyperplane than the other sheet. Such diagrams are depicted in the right of Figure 1.

Let us review Banchoff's formula. We refer to $[1,2]$ for more details. If a projection of a knotted surface is an immersion ( i.e., without branch points,) then it can be pushed off of itself in the projection direction to get a disjoint copy $F_{1}$. In general this can be done for any projection except near neighborhoods of isolated branch points. At a branch point, such a disjoint push off can be 
extended to a transverse push off so that it has a transverse double intersection point in the neighborhood of the preimage of a branch point. Thus each branch point contributes \pm 1 to the normal Euler number. The sign is determined by the crossing information near each branch point. We fix a convention that the crossing information depicted in the right of Figure 1 (C) receives a negative sign, and the mirror image of this Figure receives a positive sign.

In this paper we relate the normal Euler numbers to the number of signed triple points. A generic projection divides 3-space into regions. Thus we can give a checker-board colorings (black and white) to the regions so that the adjacent regions receive the opposite colors. We use the checker-board coloring of the regions divided by a generic projection to define the signs of triple points.

In Section 2, we define the signs of triple points and branch points of projections using such colorings together with the crossing information. In Section 3, we state our formula and give a proof. Our proof is similar to the one we used to prove Whitney's congruence between normal Euler numbers and the genus of embedded surfaces in [2].

We remark here that the idea behind Banchoff's formula is a duality between characteristic classes and singularities of generic maps [6]. Such a duality is proved for normal Euler classes for embedded 3-manifolds in 5-space in [4]. Our formula in this paper suggests such a duality involving both singularities and multiple point sets.

\section{Signs FOR TRIPLE POINTS AND BRANCH POINTS}

In this section, signs for triple points and branch points on the projections of knotted surfaces are defined using checker-board colorings.

Let $F$ be a knotted surface in 4 -space and $p(F)$ its generic projection where $p: \mathbf{R}^{4} \rightarrow \mathbf{R}^{3}$ is the projection map.

There is a checker-board coloring for the domains divided by $p(F)$ such that each domain is colored either black or white and adjacent regions (that is, regions sharing the same face) have different colorings. Fix such a coloring.

First we define signs for triple points. There are eight regions near a triple point. Pick a black region, $B$, among four of them. Let $\vec{v}_{1}, \vec{v}_{2}$ and $\vec{v}_{3}$ be the normal vectors of three sheets in $B$ pointing into $B$. Recall that these sheets are disjointly embedded in 4 -space as the top, middle, and bottom sheets. Choose $\vec{v}_{1}, \vec{v}_{2}$ and $\vec{v}_{3}$ to be the normals to the top, middle, and bottom sheets, respectively. Thus the vectors $v_{1}, v_{2}$, and $v_{3}$ are the normal vectors such that (1) they are normal to the top, middle, and bottom sheets respectively, and (2) they lie in $B$ pointing into $B$. See Figure 2. Define the triple point to be positive if the orientation of 3-space defined by the ordered triple $\left(\vec{v}_{1}, \vec{v}_{2}, \vec{v}_{3}\right)$ matches the originally fixed orientation of the 3 -space, and negative otherwise. Figure 2 depicts such vectors and checkerboard coloring at the neighborhood of a triple point. In the right hand convention this is a negative triple point. This definition depends upon the fixed orientation of the 3-space and the coloring, but it does not depend upon the choice of the black region. Let $B^{\prime}$ be another black region. Then exactly two of the normal vectors change their directions and the other one does not. Hence the orientation defined by the new normals with respect to $B^{\prime}$ is the same as the old one. Thus each triple point has a well-defined sign. 


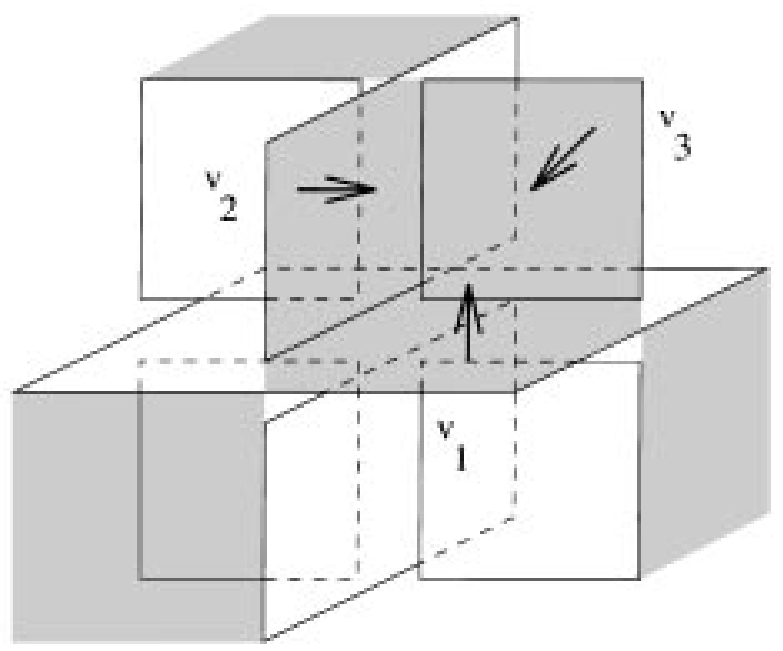

FigURE 2. Normal vectors and checker-board coloring

Next we consider the branch points. Recall that the signs of branch points are defined by Banchoff [1], where the connection to the normal Euler numbers is studied, and are also used in [2]. Such signs were defined using the crossing information near each branch point on projections of knotted surfaces. The index of the branch points was defined and used by Coghlan [7] to generalize Banchoff's formula. Such an index is also considered by Szücs [8]. Here, following Coghlan, we define the color of branch points.

In the neighborhood of a branch point $p(F)$ looks like the cone on the figure eight. Thus the checker-board coloring gives either black or white inside this figure eight. We call the former a black branch point and the latter a white branch point.

We use the following notation: $T_{+}$(resp. $\left.T_{-}\right)=$the number of the positive (resp. negative) triple points, $B_{+}$(resp. $B_{-}$) $=$the number of the positive (resp. negative) black branch points, $W_{+}$(resp. $W_{-}$) $=$the number of the positive (resp. negative) white branch points.

Further define the following numbers: $T=T_{+}-T_{-}, B=B_{+}-B_{-}$and $W=$ $W_{+}-W_{-}$.

In the next section we present a new formula involving these numbers and the normal Euler numbers.

\section{A NEW FORMUla FOR NORMAL EULER NUMBERS}

Denote by $e(F)$ the normal Euler number of $F$. Banchoff [1] proved that $e(F)$ is equal to the number of positive branch points on a generic projection $p(F)$ minus the number of negative branch points. In this section we prove our main result generalizing his formula:

\section{Theorem 3.1.}

$$
T+W+2 B=(3 / 2) e(F) .
$$

Proof. Figure 3 illustrates the fact that a triple point is replaced by a pair of branch points by adding a 1-handle. In fact this smoothing is realized as a 1-handle addition 


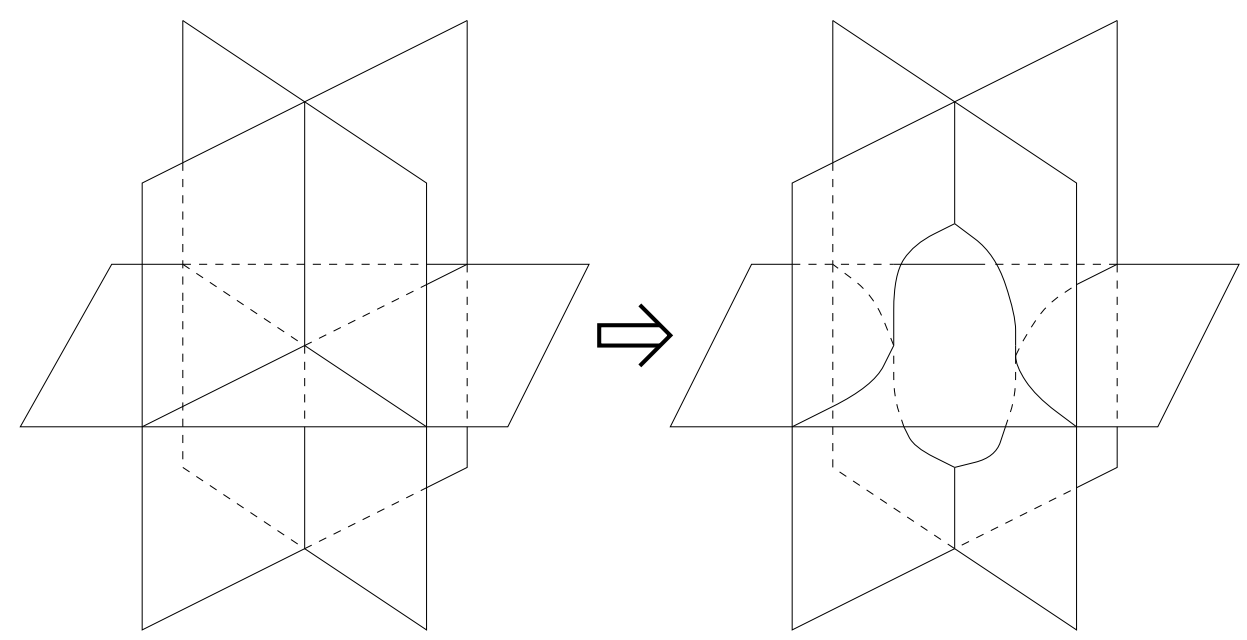

FiguRE 3. Smoothing a triple point

in 4-space so that the resulting surface is a projection of a new embedded surface in 4-space [2].

More specifically, the three sheets of surface that intersect at a triple point can be labeled top, middle, and bottom, and these indicate the relative position of the surfaces with respect to the projection direction. In order to be realized as an embedded surface, a hollow 1-handle is attached between the top and middle or between the middle and bottom sheet. If we perform this smoothing at a triple point on a projection of a knotted surface $F$, then we obtain a new knotted surface $F^{\prime}$ such that (1) $F^{\prime}$ is obtained by adding a 1-handle to $F$, and (2) the projection $F^{\prime}$ coincides with the projection $p(F)$ except in the neighborhood of the triple point and the smoothing described above is performed in this neighborhood.

We compute the changes of the numbers of signed triple points and branch points by this smoothing. Since a triple point is replaced by a pair of branch points by the smoothing, we have the following cases of changes among signed triple points and branch points:

(a) A replacement of $\{$ a positive triple point $\}$ by $\{$ a pair of a negative white branch point and a positive black branch point $\}$.

(b) A replacement of $\{$ a negative triple point $\}$ by $\{$ a pair of a positive white branch point and a negative black branch point $\}$.

Whence the integer

$$
T+W+2 B
$$

does not change by this smoothing ( $i$. e., this integer for $F$ and that of $F^{\prime}$ coincide).

Notice here that the normal Euler number does not change by 1-handle additions: $e(F)=e\left(F^{\prime}\right)$.

Now to a given projection $p(F)$ of a knotted surface $F$, perform the triple point smoothings at all the triple points. Such smoothings do not affect the integer $T+W+2 B$.

After triple point smoothings, we may have double loops (closed circles) of double points. For such a loop, perform the same smoothing as described above to get an 
arc of double points. (Simply ignore the horizontal sheet in Figure 3 in this case.) Then we obtain a pair of branch points of the same color and opposite signs. Note that this smoothing is also realized by a 1-handle addition to obtain a new knotted surface with the same normal Euler number.

Thus after these smoothings we obtain a new surface such that both the integer $T+W+2 B$ and the normal Euler number are the same as those of the original surface. The new surface has a projection only with double point arcs.

There are two types of double point arcs: the neighborhood is homeomorphic to (1) an annulus (called an $a$-arc), or (2) a Möbius band (called an $m$-arc).

The ends of an $a$-arc have opposite signs and the same color. Neither $T+W+2 B$ nor $e(F)$ changes when the $a$-arcs are replaced with disks. Let $F^{\prime \prime}$ denote the surface that results and that has only $m$-arcs.

The ends of an $m$-arc have the same sign and opposite colors. Call an $m$-arc positive if it terminates with both positive points, or negative if it terminates with negative points. Let $p$ denote the number of positive $m$-arcs and $n$ denote the number of negative $m$-arcs. Then $e\left(F^{\prime \prime}\right)=2(p-n)$.

If a positive $m$-arc is replaced by a disk $W+2 B$ changes to $W+2 B-3$. If a negative $m$-arc is replaced by a disk $W+2 B$ changes to $W+2 B+3$. After all the $m$-arcs are removed, $W+2 B=0$. So originally, $W+2 B=3(p-n)$. The theorem follows.

Corollary 3.2. If $F$ is an oriented embedded surface in 4-space, then $T(F)=0$.

Remark 3.3. This remark is due to Prof. Murasugi. If the checker-board coloring is changed, then the signs of the triple points change. The colors of the branch points also change. Thus the formula

$$
-T+B+2 W=(3 / 2) e(F)
$$

also holds. Banchoff's formula,

$$
B+W=e(F)
$$

is recovered by adding these two together. Alternately, we get

$$
W-T=T+B=(1 / 2) e(F),
$$

and

$$
T=(1 / 2)(W-B) \text {. }
$$

Remark 3.4. If we choose the white regions to determine the signs of triple points, or if we change the sign convention for branch points (but not both), then the dual formula $T+2 W+B=(3 / 2) e(F)$ holds, and similar consequences can be drawn.

\section{ACKNOWLEDGEMENTS}

We are grateful to Professors Cameron Gordon, Kunio Murasugi, Makoto Sakuma, and Shuji Yamada for valuable comments.

\section{REFERENCES}

[1] Banchoff, T. F., Double Tangency Theorems for Pairs of Submanifolds, in Geometry Symposium Utrecht 1980 ed. Looijenga, Seirsma, and Takens, LNM v. 894, Springer-Verlag (1981), 26-48. MR 83h:53005

[2] Carter, J. Scott, and Saito, Masahico, Canceling Branch Points on the Projections of Knotted Surfaces in 4-space, Proc. of the AMS. 116, No 1 (Sept 1992), 229-237. MR 93i:57029 
[3] Carter, J. Scott, and Saito, Masahico, Reidemeister Moves for Surface Isotopies and Their Interpretation as Moves to Movies, J. of Knot Theory and its Ramifications 2, 251-284. MR 94i: 57007

[4] Carter, J. Scott, and Saito, Masahico, 4-dimensional projections of 3-manifolds in 5-space, preprint.

[5] M. Golubitsky, and V. Guillemin, Stable mappings and their singularities, Graduate texts in math. vol. 14, Springer-Verlag, 1973. MR 49:6269

[6] S. E. Cappel and J. L. Shaneson, An introduction to embeddings, immersions and singularities in codimension two, Proc. Sympos. Pure Math. vol. 32, AMS, Providence, RI, 1978. MR 80e: 57013

[7] Coghlan, Leslie, Private correspondences.

[8] A. Szücs, Surfaces in $\mathbf{R}^{3}$, Bull. London Math. Soc. 18 (1986), 60-66. MR 88a:57060

Department of Mathematics and Statistics, University of South Alabama, Mobile, Alabama 36688

E-mail address: carter@mathstat.usouthal.edu

Department of Mathematics, University of South Florida, Tampa, Florida 33620

E-mail address: saito@math.usf.edu 